C.D. MINDA

KODAI MATH. J.

2 (1979), 420-426

\title{
THE AUMANN-CARATHÉODORY RIGIDITY CONSTANT FOR DOUBLY CONNECTED REGIONS
}

\author{
By CARL DAVID Minda ${ }^{1}$
}

1. Introduction. Suppose $G$ is a nonsimply connected region in the extended complex plane $\boldsymbol{P}$ such that $\boldsymbol{P} \backslash G$ contains at least three points and $a \in$ $G$. Aumann and Carathéodory [3] proved that there is a constant $\Omega=\Omega(G, a)$; $0 \leqq \Omega<1$, such that if $f$ is any analytic self-mapping of $G$ fixing $a$ and $f$ is not a conformal automorphism of $G$, then $\left|f^{\prime}(a)\right| \leqq \Omega$. In this paper we will explicitly determine the Aumann-Carathéodory rigidity constant for proper doubly connected regions and identify all extremal functions. The value of the constant and the form of the extremal functions were rather implicitly determined by Heins [4] and Hervé [6]. Our work is simultaneously more explicit and more elementary; it is based upon the use of covering surfaces.

Since the Aumann-Carathéodory constant is a conformal invariant, it suffices to consider the case of an annulus because every proper doubly connected region is conformally equivalent to an annulus. Fix $R>1$. Throughout this paper $A=\{z: 1 / R<|z|<R\}$. Take $a \in A$ and let $\mathscr{F}=\mathscr{F}(A, a)$ be the family of all analytic functions $f: A \rightarrow A$ such that $f(a)=a$ and $f$ is not a conformal automorphism of $A$. In this paper we shall find

$$
\Omega=\Omega(A, a)=\max _{f \in \mathscr{F}}\left|f^{\prime}(a)\right|
$$

and the extremal functions.

2. Expressing $\Omega$ as the quotient of two conformally invariant metrics. The following result will enable us to express the Aumann-Carathéodory rigidity constant for an annulus as the quotient of two basic conformally invariant metrics.

ANNulus THEOREM. Let $\gamma$ be a generator of the fundamental group of $A$. Suppose $f$ is an analytic self-mapping of $A$ such that $f \circ \gamma$ is freely homotopic to $\gamma^{n}$ for some integer $n$. Then $n=0, \pm 1$. Moreover, $|n|=1$ if and only if $f$ is $a$ conformal automorphism of $A$ and $n=0$ if and only if $f$ is homotopic to a constant.

Received September 8, 1978

${ }^{1}$ Research supported in part by National Scinece Foundation Grant MCS78-02662. 
This theorem has a variety of different proofs ([7], [8], [9], [10], [11], [13]). In view of the covering surface theme of this paper we shall give yet another proof. This proof is essentially due to Heins [5] and shows that the annulus theorem should properly be traced back to his work.

Proof of the annulus theorem. Without loss of generality we may assume that $n \geqq 0$; if not, then replace $f$ by $1 / f$. Let $H=\{z: \operatorname{Re}(z)>0\}$ and suppose that $\pi: H \rightarrow A$ is an analytic universal covering projection. By an appropriate choice of $\pi$ we can assure that the group of cover transformations is the cyclic group generated by $T(z)=\lambda z$, where $\lambda>1$. Fix $a \in A$ and define $b=f(a)$. Select points $\tilde{a}, \tilde{b} \in H$ with $\pi(\tilde{a})=a, \pi(\tilde{b})=b$. Then there is a unique analytic function $\tilde{f}: H \rightarrow$ $H$ such that $\tilde{f}(\tilde{a})=\tilde{b}$ and $\pi \circ \tilde{f}=f \circ \pi$. Since $f \circ \gamma$ is freely homotopic to $\gamma^{n}$, it follows that $\tilde{f} \circ T=T^{n} \circ \tilde{f}$; that is, $\tilde{f}(\lambda z)=\lambda^{n} \tilde{f}(z)$ for all $z \in H$.

First, we show that $n$ is either 0 or 1 . Let $c$ be the angular derivative of $\tilde{f}$ at $\infty$, then $0 \leqq c<\infty$ by the Julia-Carathéodory theorem. Fix any $z \in H$, then

$$
\lim _{k \rightarrow \infty} \frac{\tilde{f}\left(\lambda^{k} z\right)}{\lambda^{k} z}=c .
$$

But the functional equation satisfied by $\tilde{f}$ shows that $\tilde{f}\left(\lambda^{k} z\right)=\lambda^{n k} \tilde{f}(z)$, so that

$$
c=\frac{\tilde{f}(z)}{z} \lim _{k \rightarrow \infty} \lambda^{(n-1) k} \text {. }
$$

Since $\lambda^{(n-1) k}$ is unbounded if $n \geqq 2$, we obtain a contradiction unless $n=0,1$. If $n=1$, then we obtain $\tilde{f}(z)=c z$ and $c$ must be positive. This implies that $f$ is a rotation, so a conformal automorphism of $A$.

When $n=0, f \circ \gamma$ is freely homotopic to a point, so $f$ lifts to an analytic function $\tilde{f}: A \rightarrow H$ such that $\pi \circ \tilde{f}=f$. Clearly, $\tilde{f}$ is homotopic to a constant, so the same is true of $f$.

Thus, if $f \in \mathscr{F}$, then the annulus theorem shows that $f$ is homotopic to a constant. In particular, $f$ induces the trivial homomorphism on the fundamental group. Let $\pi: B \rightarrow A$ be the unique analytic universal covering projection of the unit ball $\boldsymbol{B}$ onto $A$ such that $\pi(0)=a$ and $\pi^{\prime}(0)>0$. Then each $f \in \mathscr{F}$ lifts to a unique analytic function $\tilde{f}: A \rightarrow \boldsymbol{B}$ such that $\tilde{f}(a)=0$ and $\pi \circ \tilde{f}=f$. Conversely, if $\tilde{f}: A \rightarrow \boldsymbol{B}$ is an analytic function with $\tilde{f}(a)=0$, then $\pi \circ \tilde{f} \in \mathscr{I}$. Let $\beta=\beta(A, a)$ be the family of all analytic functions $\tilde{f}: A \rightarrow B$ which satisfy $\tilde{f}(a)=0$. Then $\mathscr{F}=\{\pi \circ \tilde{f}: \tilde{f} \in \beta\}$ and

$$
\begin{aligned}
\Omega & =\max _{\widetilde{f} \in \beta}\left|(\pi \circ \tilde{f})^{\prime}(a)\right| \\
& =\pi^{\prime}(0) \max _{\widetilde{f} \in \beta}\left|\tilde{f}^{\prime}(a)\right| .
\end{aligned}
$$

Now, $\pi^{\prime}(0)=1 / \lambda(a)$, where $\lambda(z)|d z|$ is the hyperbolic metric on $A$ and

$$
c_{B}(a)=\max _{\widetilde{f} \in \beta}\left|\tilde{f}^{\prime}(a)\right|,
$$

where $c_{B}(z)|d z|$ is the metric on $A$ induced by analytic capacity. Thus, 


$$
\Omega=c_{B}(a) / \lambda(a) \text {. }
$$

Note that $f \in \mathscr{F}$ is extremal for $\Omega$ if and only if the associated function $\tilde{f} \in \beta$ is extremal for $c_{B}(a)$.

3. The Ahlfors function for an annulus. Ahlfors [2] showed that the extremal function $h$ in $\beta$ which attains the value $c_{B}(a)$ is unique up to rotation and $h$ is a two-sheeted branched covering of $A$ onto $\boldsymbol{B}$. Actually, Ahlfors determined the extremal function for analytic capacity for regions of finite connectivity. For the case of an annulus it is possible to give an elementary proof. We begin by studying two-sheeted branched coverings of $A$ onto $\boldsymbol{B}$.

Proposition. Fix $a, b \in A$. There is a two-sheeted branched covering $f: A \rightarrow$ $\boldsymbol{B}$ with $f(a)=0=f(b)$ if and only if $|a b|=1$. (In case $a=b$ we require that $f$ also have a branch point of a.) The function $f$ is unique up to rotation and $f^{\prime}$ has a simple zero at $\pm \sqrt{a b}$ and no other zeros.

Proof. First, suppose that $f: A \rightarrow \boldsymbol{B}$ is a two-sheeted branched covering with $f(a)=f(b)=0$. Observe that $|f|=1$ on $\partial A$. Now, $\log |f|$ is negative and harmonic on $c l(A)$ except for the points $a, b$ where it has logarithmic singularities and vanishes on $\partial A$. Therefore, $-\log |f(z)|=g(z, a)+g(z, b)$, where $g(z, a), g(z, b)$ is the Green's function for $A$ with singularity at $a, b$, respectively. Recall that

$$
\omega(z)=\frac{\log |z|+\log R}{2 \log R}
$$

is the harmonic measure of $\gamma=\{z:|z|=R\}$ with respect to $A$. The PoissonGreen integral formula for an annulus gives

$$
-2 \pi \omega(a)=\int_{\partial A} \omega(z) * d g(z, a)=\int_{r} * d g(z, a) .
$$

Of course, the same result holds with a replaced by $b$. Since $f$ maps each oriented contour of $\partial A$ onto $\partial \boldsymbol{B}$ traversed once in the positive direction, we obtain

$$
\int_{r} * d \log |f|=2 \pi
$$

Consequently,

$$
1=\omega(a)+\omega(b)=\frac{\log |a b|+2 \log R}{2 \log R} .
$$

or $|a b|=1$. This establishes the necessity of the condition.

Conversely, assume $a, b \in A$ and $|a b|=1$. Then the function $u(z)=g(z, a)$ $+g(z, b)$ is positive and harmonic in $c l(A)$ except for logarithmic singularities at $a, b$ and vanishes on $\partial A$. Let $\delta(a), \delta(b)$ be small, positively oriented circles 
centered at $a, b$, respectively. Then

$$
\int_{\delta(a)} * d u=-2 \pi=\int_{\delta(b)} * d u
$$

If $|a b|=1$, then our preceding work shows that

$$
\int_{r} * d u=-2 \pi[\omega(a)+\omega(b)]=-2 \pi .
$$

Since $\delta(a), \delta(b), \gamma$ form a homology basis for $\operatorname{cl}(A) \backslash\{a, b\}$, this implies that all periods of $* d u$ are an integer multiple of $2 \pi$. If $u *$ is the multiple-valued harmonic conjugate of $u$, then $f=\exp [-(u+\imath u *)]$ is a single-valued analytic function in $A$ with zeros at $a$ and $b$. (A simple zero at each point if $a \neq b$ and a double zero at $a$ if $a=b$.) Also, $|f|=1$ on $\partial A$ and $f$ maps $A$ into $B$. The boundary correspondence of $f$ implies that $f$ is an $n$-sheeted branched covering of $A$ onto $\boldsymbol{B}$ for some positive integer $n$. The fact that $f$ has exactly two zeros shows that $n=2$. Hence, $|a b|=1$ is sufficient for the existence of a twosheeted covering $f: A \rightarrow B$ with $f(a)=f(b)=0$.

That two points $a, b \in A$ with $|a b|=1$ determine the two-sheeted covering $f: A \rightarrow \boldsymbol{B}$ up to rotation is elementary; just apply the maximum principle to the quotient of two such coverings.

Finally, we locate the zeros of $f^{\prime}$. Since $|a b|=1, T(z)=a b / z$ is a conformal automorphism of $A$ which permutes $a$ and $b$. Also, $T$ fixes the two points $\pm \sqrt{a b}$. Now, $f \circ T$ is a two-sheeted branched covering of $A$ onto $\boldsymbol{B}$ sending $a$ and $b$ to 0 . Thus, $f \circ T=\lambda f$ for some $\lambda$ with $|\lambda|=1$. Since $T \circ T$ is the identity, we obtain $\lambda^{2}=1$, or $\lambda= \pm 1$. If $f \circ T=-f$, then $f( \pm \sqrt{a b})=0$ which implies $a \cdot b=$ $\sqrt{a b} \cdot(-\sqrt{a b})$, a contradiction. Thus, $f \circ T=f$. From $T^{\prime}( \pm \sqrt{a b})=-1$, we obtain $f^{\prime}( \pm \sqrt{a b})=0$.

THEOREM. Let $h: A \rightarrow \boldsymbol{B}$ be the unique two-sheeted branched coverng with $h(a)=0=h(-1 / \bar{a})$ and $h^{\prime}(a)>0$. Then $\left|\tilde{f}^{\prime}(a)\right| \leqq h^{\prime}(a)$ for any $\tilde{f} \in B$ and equality holds if and only if $\tilde{f}=\lambda h$ for some $\lambda$ with $|\lambda|=1$.

Proof. Observe that the quotient $g=\tilde{f} / h$ is analytic in $A$ except possibly for a simple pole at $-1 / \bar{a}$. Also, $\limsup _{z \rightarrow \zeta}|g(z)| \leqq 1$ for any $\zeta \in \partial A$. Therefore, a lemma of Robinson [12] as refined by Âbe [1] implies that $|g(a)| \leqq 1$ and equality holds if and only if $g$ is a constant function of modulus 1 . Since $g(a)=\tilde{f}^{\prime}(a) /$ $h^{\prime}(a)$, this establishes the inequality.

4. Summary. Let us now gather together our results.

THEOREM. Let $\pi: B \rightarrow A$ be the unque analytic unversal coverng projection with $\pi(0)=a$ and $1 / \lambda(a)=\pi^{\prime}(0)>0$. Let $h: A \rightarrow B$ be the unique two-sheeted branched covering with zeros at $a,-1 / \bar{a}$ and $c_{B}(a)=h^{\prime}(a)>0$. Then the value region $\left\{f^{\prime}(a)\right.$ : $f \in \mathscr{F}\}$ is the closed ball with radius $\Omega=c_{B}(a) / \lambda(a)$ and center $0 . f_{\lambda}=\pi \circ(\lambda h),|\lambda|=1$, 
is the unique function in $\mathscr{F}$ which satısfies $f_{\lambda}^{\prime}(a)=\lambda \Omega$. Moreover, the only zeros of $f_{\lambda}^{\prime}$ are simple zeros at $\pm \sqrt{-a / \bar{a}}$.

We have already established most of the assertions in this theorem; only the statement about the value region needs consideration. The functions $f_{\lambda}$, where we now permit $|\lambda| \leqq 1$, show that the value region is the closed ball with radius $\Omega$ and center 0 . Thus, the extremal functions for the Aumann-Carathéodory rigidity constant for a proper doubly connected region are infinite-sheeted branched coverings of the region onto itself with exactly two branch points.

5. Numerical evaluation of $\Omega$. In order to evaluate the Aumann-Carathéodory rigidity constant we shall separately calculate the hyperbolic metric and the analytic capacity. By making use of an explicit analytic universal covering projection of the unit ball onto $A$, it is straightforward to show that

$$
\lambda(a)=\frac{\pi}{4 \log R} \cdot \frac{1}{|a| \cos \left(\frac{\pi}{2} \cdot \frac{\log |a|}{\log R}\right)}
$$

The calculation of the analytic capacity is more involved. We begin by expressing the Ahlfors function in terms of theta functions. This is analogous to work of Robinson [12] and Abe [1]. Let

$$
\theta(z)=\theta\left(z, 1 / R^{2}\right)=\prod_{n=1}^{\infty}\left(1-1 / R^{4 n}\right)\left(1+z / R^{4 n-2}\right)\left(1+1 / z R^{4 n-2}\right)=\sum_{-\infty}^{\infty} R^{-2 n^{2}} z^{n} .
$$

(This is the usual theta function with $q$ replaced by $1 / R^{2}$.) $\theta$ has a simple zero at the points $z=-1 / R^{4 n-2}, n=0, \pm 1, \cdots$ and no other zeros. The following functional equations are satisfied by $\theta$.

$$
\theta(z)=\overline{\theta(\bar{z})}=\theta(1 / z)=\left(z / R^{2}\right) \theta\left(z / R^{4}\right)
$$

From these facts we conclude that $\theta\left(-z / R^{2} a\right)$ has a simple zero at $a$ and no other zeros in $A$. Also, $\theta(-\bar{a} z)$ has no zero in A. Therefore, $f(z ; a)=\theta(-z /$ $\left.R^{2} a\right) / \theta(-\bar{a} z)$ is analytic in $A$ with $a$ simple zero at $a$. By making use of the functional equations for $\theta$ it is elementary to show that $f(z ; a)$ has modulus 1 on $|z|=R$ and modulus $R|a|$ on $|z|=1 / R$. Similar results hold for the function $f(z ;-1 / \bar{a})$. Therefore, the function $h(z)=(z / R) f(z ; a) f(z ;-1 / \bar{a})$ is analytic in $A$, has modulus 1 on $\partial A$ and has simple zeros at $a,-1 / \vec{a}$. Thus, up to a possible rotation $h$ is the Ahlfors function for $A$ with respect to the base point a. We shall see that $h^{\prime}(a)>0$ so $h$ is the Ahlfors function.

$$
\begin{aligned}
c_{B}(a) & =h^{\prime}(a)=(a / R) f^{\prime}(a ; a) f(a ;-1 / \bar{a}) \\
& =\frac{a}{R} \cdot \frac{-1}{R^{2} a}-\theta\left(-|a|^{2}\right) \\
& =\frac{\theta\left(|a|^{2} / R^{2}\right)}{\theta(1)} \\
& =\frac{\left(1+1 /|a|^{2}\right)}{R} \prod_{n=1}^{\infty} \frac{\left(1-1 / R^{4 n}\right)^{2}\left(1+|a|^{2} / R^{4 n}\right)\left(1+1 /|a|^{2} R^{4 n}\right)}{\left(1+1 / R^{4 n-2}\right)^{2}\left(1-|a|^{2} / R^{4 n-2}\right)\left(1-1 /|a|^{2} R^{4 n-2}\right)}
\end{aligned}
$$


Our expressions for $c_{B}(a)$ and $\lambda(a)$ reveal that

$$
\lim _{a \rightarrow \partial \Delta A} \frac{c_{B}(a)}{\lambda(a)}=1
$$

so that the Aumann-Carathéodory constant is not uniformly less than one in $A$. Also, for $|a|=1$ the expression for $\Omega$ simplies as follows:

$$
\begin{aligned}
\Omega(A, a) & =\frac{c_{B}(a)}{\lambda(a)}=\frac{4 \log R}{\pi} \cdot \frac{2}{R} \prod_{n=1}^{\infty} \frac{\left(1-1 / R^{8 n}\right)^{2}}{\left(1-1 / R^{8 n-4}\right)^{2}} \\
& =\frac{8 \log R}{\pi R} \prod_{n=1}^{\infty}\left(1-1 / R^{16 n}\right)^{2}\left(1+1 / R^{8 n-4}\right)^{2} .
\end{aligned}
$$

6. Extension to regions of higher connectivity. Let $G$ be a nonsimply connected region in the extended complex plane $\boldsymbol{P}$ such that $\boldsymbol{P} \backslash G$ contains at least three points. Fix $a \in G$ and let $\mathscr{F}=\mathscr{F}(G, a)$ be the family of all analytic self-mappings of $G$ which fix $a$ and are not conformal automorphisms of $G$. The Aumann-Carathéodory constant is

$$
\Omega=\Omega(G, a)=\max _{f \in \mathscr{I}}\left|f^{\prime}(a)\right| .
$$

Let $\mathscr{F}_{0}$ be the subfamily of $\mathscr{F}$ consisting of all functions which are homotopic to a constant and set

$$
\Omega_{0}=\Omega_{0}(G, a)=\max _{f \in \mathscr{F}_{0}}\left|f^{\prime}(a)\right| .
$$

Clearly, $\Omega_{0} \leqq \Omega$. Let $\pi: B \rightarrow G$ be the unique analytic universal covering projection satisfying $\pi(0)=a$ and $\pi^{\prime}(0)>0$. Then $\mathscr{F}_{0}=\{\pi \circ \tilde{f}: \tilde{f} \in \beta\}$, where $\beta=\beta(G, a)$ is the family of all analytic functions $\tilde{f}: G \rightarrow \boldsymbol{B}$ that satisfy $\tilde{f}(a)=0$. Therefore,

$$
\Omega_{0}=\pi^{\prime}(0) \max _{\widetilde{f} \in \beta}\left|f^{\prime}(a)\right|=c_{B}(a) / \lambda(a),
$$

where $c_{B}(z)|d z|$ is the analytic capacity metric on $G$ and $\lambda(z)|d z|$ is the hyperbolic metric on $G$. The extremal functions for $\Omega_{0}$ are $\pi \circ(\lambda h),|\lambda|=1$, where $h: G \rightarrow \boldsymbol{B}$ is the Ahlfors function, and the value region $\left\{f^{\prime}(a): f \in \mathscr{F}_{0}\right\}$ is the closed ball with radius $\Omega_{0}$ and center 0 . Thus, in the general case we only find that $c_{B}(a) / \lambda(a)$ is a lower bound for the Aumann-Carathéodory rigidity constant.

\section{REFERENCES}

[1] H. ABE, On some analytic functions in an annulus, Kōdai Math. Sem. Rep., 10 (1958), 38-45.

[2] L.V. Ahlfors, Bounded analytic functions, Duke Math. J., 14 (1947), 1-11.

[3] G. Aumann and C. Carathéodory, Ein Satz über die konforme Abbildung mehrfach zusammenhängender Gebiete, Math. Ann., 109 (1934), 756-763. 
[4] M. Heins, Extremal problems for functions analytic and single-valued in a doubly connected region, Amer. J. Math., 62 (1940), 91-106.

[5] M. Heins, On the iteration of functions, which are analytic and single valued in a given multiply connected region, Amer. J. Math., 63 (1941), 461-480.

[6] M. Hervé, Quelques proprieties des transformations intérieures d'un domain borné, Ann. Sci. Ecole Norm. Sup., Ser. 368 (1951), 125-184.

[7] H. HubER, Über analytische Abbildungen von Ringgebieten in Ringgebiete, Compos. Math., 9 (1951), 161-168.

[8] J.A. Jenkins, Some results related to extremal length, Annals of Math. Studies no. 30, Princeton Univ. Press, 1953.

[9] S. Kobayashi, Hyperbolic manifolds and holomorphic mappings, Pure and Applied Mathematics, vol. 2, Marcel Dekker, New York, 1970.

[10] H. L. Landau and R. Osserman, On analytic mappings of Riemann surfaces, J. Anal. Math., 7 (1959-60), 249-279.

[11] E. REICH, Elementary proof of a theorem on conformal rigidity, Proc. Amer. Math. Soc., 17 (1966), 644-645.

[12] R. M. Robinson, Analytic functions in circular rings, Duke Math. J., 10 (1943), 341-354.

[13] M. Schiffer, On the modulus of doubly-connected domains, Quart, J. Math., 17 (1946), 197-213.

Departiment of Mathematics

UNIVERSITY OF CinCINNATI

Cincinnati, Ohio 45221 